\title{
ESOFAGECTOMIA DE RESGATE APÓS QUIMIORRADIOTERAPIA RADICAL EXCLUSIVA: RESULTADOS DO DEPARTAMENTO DE CIRURGIA ABDÔMINOPÉLVICA DO INSTITUTO NACIONAL DE CÂNCER
}

\author{
SALVAGE ESOPHAGECTOMY AFTER EXCLUSIVE RADICAL \\ CHEMORADIOTHERAPY: RESULTS OF THE ABDOMINOPELVIC SURGERY \\ DEPARTMENT IN THE NATIONAL CANCER INSTITUTE
}

\author{
Carlos Eduardo Pinto, TCBC-RJ'1 ; Jurandir Almeida Dias, TCBC-RJ'2; \\ Eduardo Amaral Moura Sá AsCBC-RJ ${ }^{3}$; André Luís Lopes Carvalho, ACBC-BA ${ }^{4}$
}

\begin{abstract}
RESUMO: Objetivo: Relatar a experiência e os resultados da Seção de Cirurgia Abdôminopélvica do Instituto Nacional de Câncer (INCA) com a esofagectomia de resgate em paciente portador de câncer de esôfago recidivado após tratamento quimiorradioterápico exclusivo. Método: Foram analisados retrospectivamente 14 pacientes portadores de câncer de esôfago recidivado e que foram submetidos à esofagectomia de resgate entre março de 1999 e maio de 2006. Todos os pacientes incluídos no estudo receberam tratamento primário quimiorradioterápico radical exclusivo conforme protocolo RTOG 85-01 e apresentaram persistência ou recidiva de doença. Resultados: A idade média foi de 63 anos (39-72 anos). Oito pacientes eram do sexo masculino e seis pacientes do sexo feminino. Nove pacientes apresentavam o tumor localizado no esôfago médio e cinco pacientes apresentavam doença no esôfago distal, sendo carcinoma epidermóide em 12 pacientes e adenocarcinoma em dois pacientes. A mediana do tempo cirúrgico foi de 305 minutos (240-430 minutos). A ressecção completa do tumor (cirurgia R0) foi realizada em 13 pacientes e somente um paciente apresentou doença residual macroscópica em ápice pulmonar. A morbidade total da série foi de $69,2 \%$. A mortalidade operatória foi zero (todos os pacientes evoluíram para alta hospitalar). Conclusão: A esofagectomia de resgate demonstrou-se factível tecnicamente porém apresenta elevada morbidade operatória. Esta modalidade cirúrgica corresponde atualmente ao melhor tratamento disponível para se obter cura nos casos de tumor recidivado ou que tenho persistido com doença após quimiorradioterapia radical exclusiva. Todos os outros tipos de tratamento são considerados paliativos e com resultados de sobre-vida desapontadores (Rev. Col. Bras. Cir. 2007; 34(1): 16-20).
\end{abstract}

Descritores: Esofagectomia; Neoplasias esofágicas; Terapia combinada; Quimioterapia; Radioterapia; Instituições de saúde.

\section{INTRODUÇÃO}

O câncer de esôfago corresponde a 1-2\% dos tumores malignos e a sétima causa de morte por câncer nos EUA. No Brasil é a nona neoplasia mais freqüente, sendo a sexta causa de morte por câncer $^{1-3}$. Esta neoplasia apresenta um grande desafio quanto ao seu tratamento devido à alta mortalidade e morbidade operatória. Seu diagnóstico frequentemente é feito em estágios avançados, o que dificulta muito o tratamento adequado e torna bastante desfavorável o prognósti$\mathrm{co}^{4}$.

A ressecção cirúrgica tornou-se o tratamento considerado padrão ouro para o câncer de esôfago a partir da década de 40, com índices globais de cura variando de 15 a $40 \%$ (sendo menos de 5\% para os estágios avançados) ${ }^{4-6}$. Há algumas décadas a mortalidade operatória girava em torno de $10 \%$, sendo hoje menos de $5 \%$ em centros de referência ${ }^{7,8}$. Devido à baixa efetividade da esofagectomia em termos curativos, novas modalidades terapêuticas têm sido pesquisadas geral- mente em combinação com a ressecção cirúrgica. Radioterapia, quimioterapia ou quimiorradioterapia (adjuvante e/ou neoadjuvante) ou ainda a quimiorradiação radical exclusiva fazem parte do arsenal disponível atualmente ${ }^{9}$.

Têm-se preconizado a quimiorradioterapia radical exclusiva para os pacientes portadores de carcinomas esofágicos localmente avançados (T3 ou T4) ou em pacientes sem reserva fisiológica para a esofagectomia, sendo observado uma sobrevida em cinco anos de 10-30\%. Entretanto, o controle locorregional é pobre com taxas de recidiva local relatada na literatura de 40-60\%. A esofagectomia de resgate fornece a única possibilidade de cura para pacientes selecionados com recorrência tumoral ou persistência de doença após a quimiorradioterapia exclusiva, com taxas de sobrevida em cinco anos de aproximadamente $25 \%{ }^{10}$.

Do ponto de vista teórico, a cirurgia de resgate é dotada de maior dificuldade técnica e morbimortalidade operatória, devido à alta dose de radiação aplicada no leito tumoral e ao maior intervalo de tempo entre o término do tratamento

1. Doutor e Mestre em Cirurgia pela UFRJ; Cirurgião Coordenador do Grupo de Esôfago do INCA.

2. Chefe do Serviço de Cirurgia Abdômino-pélvica do INCA.

3. Residente de Cirurgia Oncológica do INCA, ACBC.

4. Ex-Residente de Cirurgia Oncológica do INCA.

Recebido em 29/07/06

Aceito para publicação em 30/08/06

Conflito de interesses: nenhum

Fonte de financiamento: nenhuma

Trabalho realizado no Serviço de Cirurgia Abdôminopélvica do Instituto Nacional de Câncer, Rio de Janeiro - RJ. 
combinado e a cirurgia (determinando maior grau de fibrose entre as estruturas periesofagianas). Tal fato, associado ao ceticismo em relação à cura do câncer de esôfago explica a relutância de muitos cirurgiões em realizar tal operação.

Diante destes fatores o principal objetivo do presente estudo é relatar a experiência e os resultados da Seção de Cirurgia Abdôminopélvica do INCA com a esofagectomia de resgate (pós-quimiorradioterapia radical exclusiva). Este procedimento cirúrgico constitui uma novíssima modalidade terapêutica que surgiu com o advento das insurgentes formas de tratamento desenvolvidas nas últimas décadas para o câncer de esofágico e em especial a quimioradioterapia exclusiva.

\section{MÉTODO}

Foram analisados retrospectivamente 14 pacientes submetidos à esofagectomia de resgate após tratamento quimiorradioterápico radical exclusivo, no Serviço de Cirurgia Abdôminopélvica do INCA entre março de 1999 e maio de 2006. A avaliação do estudo foi feita com base nos prontuários e no programa de qualidade total da Seção de Cirurgia Abdôminopélvica. O diagnóstico de recidiva foi confirmado por biópsia endoscópica em todos os casos e os pacientes foram selecionados para o resgate cirúrgico levando em conta seu "performance status" (I ou II pela escala de Zubroid - ECOG), presença de doença ressecavél na avaliação pré-operatória e condição clinica adequada para realização do procedimento.

A indicação do tratamento primário com quimiorradioterapia exclusiva se deu para pacientes com estadiamento T3 ou T4 de acordo com a classificação TNM (AJCC/UICC) ou para pacientes que apresentavam alguma contra-indicação clinica ao tratamento operatório.

O tratamento combinado radical padrão realizado no INCA corresponde ao protocolo RTOG 85-01, a saber: Quimioterapia com Cisplatina (CDDP): $75 \mathrm{mg} / \mathrm{m}^{2}\left(1^{\circ}\right.$ dia das semanas $1,5,8$ e 11) e 5-Fluoracil: $1000 \mathrm{mg} / \mathrm{m}^{2}$ (infusão contínua nos quatro primeiros dias de cada ciclo de cisplatina) e radioterapia ( $30 \mathrm{~Gy}$ - 15 frações em três semanas com reforço de 20 Gy - 10 frações em duas semanas). Dois pacientes receberam reforço no leito tumoral com braquiterapia complementar (15 Gy) e dois pacientes submeteram-se ao tratamento combinado fora da instituição e foram referenciados ao INCA por persistência de doença restrita ao esôfago.

Todos os pacientes receberam controle endoscópico rigoroso com endoscopia a cada três meses ou em caso de alguma sintomatologia. Os pacientes considerados com persistência de doença foram aqueles que apresentaram biópsia endoscópica positiva para malignidade até três meses após o término do tratamento combinado. Eram considerados com recidiva os pacientes com biópsia positiva após três meses.

O acompanhamento dos pacientes após o término do tratamento primário e após o resgate cirúrgico foi realizado por uma equipe multiprofissional, com avaliação médica, fisioterápica e nutricional periódicas. O re-estadiamento dos pacientes candidatos ao resgate cirúrgico se deu principalmente com a tomografia computadorizada de tórax, abdome e pelve, sendo fundamental à comparação com exames executados no estadiamento primário para uma avaliação correta.

\section{RESULTADOS}

Do total de 14 pacientes, oito eram do sexo masculino e seis do sexo feminino, com idade mediana de 63 anos (3972 anos). Quanto à topografia da lesão no esôfago, o terço inferior esteve envolvido em cinco pacientes e o terço médio nos outros nove pacientes. O intervalo livre de doença pósquimioradiação variou de 1,5 a 29 meses, com média de 7,65 meses. (Tabela 1)

O tipo histológico predominante foi o carcinoma epidermóide encontrado em 12 pacientes, sendo o adenocarcinoma observado em dois pacientes. A invasão angiolinfática esteve presente em seis casos e a invasão neural em quatro casos. A média do maior diâmetro tumoral foi de 3,53 cm (1,5-6,5 m). A via cirúrgica trans-torácica foi realizada em 12 pacientes e a trans-hiatal em dois pacientes. O número de linfonodos dissecados variou de zero a 38 linfonodos (média de 13,2), sendo acometido por neoplasia em cinco pacientes (média de 1,3 linfonodos metastático na série). Em um paciente estava presente uma massa linfonodal mediastinal metastática, que foi completamente ressecada em monobloco com a peça cirúrgica. Em 13 pacientes a ressecção foi documentada como RO (sem resíduo tumoral), através da biópsia de congelação em todo o leito tumoral. Em outro caso encontramos um nódulo no ápice do pulmão direito, cuja biópsia de congelação demonstrou ausência de malignidade, porém o exame de parafina mostrou tratar de uma lesão metastática. A mediana do tempo cirúrgico foi de 305 minutos (240-430 minutos), sendo somente dois pacientes submetidos a hemotransfusão intra-operatória. Três pacientes necessitaram de ventilação mecânica prolongada (definida como mais de 24 horas), sendo 11 pacientes extubados no centro cirúrgico. A mediana do tempo de terapia intensiva foi de quatro dias (2-37 dias) e a de internação hospitalar foi de 14,4 dias (11-59 dias).

Tabela 1 - Características dos pacientes $(n=14)$.

Mediana de idade 63 anos (39-72 anos)

\section{Sexo}

Masculino

08 pacientes $(57,1 \%)$

Feminino

06 pacientes $(42,9 \%)$

Localização do tumor

$1 / 3$ médio

$1 / 3$ inferior

09 pacientes $(64,2 \%)$

05 pacientes $(35,8 \%)$

Tipo Histológico

Carcinoma epidermóide $\quad 12$ pacientes $(85,7 \%)$

Adenocarcinoma

02 pacientes $(14,3 \%)$

Ressecção cirúrgica

$\mathrm{RO}$

R1

13 pacientes $(92,8 \%)$

0

R2 01 paciente $(7,2 \%)$

Intervalo Livre de Doença

Média 7,65 meses (1,5-29)

(pós-quimiorradiação) 
Dentre as complicações técnicas, destacam-se: uma fístula anastomótica cervical, com resolução espontânea, uma deiscência de sutura do tubo gástrico que provocou mediastinite, e necessitou a retirada do mesmo e lavagem do mediastino, uma necrose do tubo gástrico por congestão venosa (sem extravasamento anastomótico), que também necessitou de exérese do tubo e uma fistula esôfago-traqueal, tratada conservadoramente.

Ocorreram também cinco casos de pneumonia, quatro casos de infecção da corrente sanguínea (bacteremia), um caso de infecção urinária, um caso de infecção do local cirúrgico, um caso de íleo paralítico,um caso de trombose venosa profunda e um caso de tromboembolismo pulmonar. A morbidade geral da série foi de $69,2 \%$. A mortalidade operatória foi zero. Todos os pacientes tiveram alta hospitalar.

O seguimento médio foi de 12,2 meses e dois pacientes foram a óbito. Um paciente faleceu em 12 meses de seguimento com recidiva locorregional (linfadenopatias mediastinais) e o outro em quatro meses também com recidiva locorregional (linfonodos cervicais) e metástase pulmonar. Doze pacientes permanecem vivos, onze sem evidência de doença locorregional e um com doença mediastinal. A sobrevida média da série foi de 12,6 meses.

\section{DISCUSSÃO}

Desde a década de 40, a esofagectomia tornou-se o tratamento padrão para o câncer de esôfago. Seus índices globais de cura variam de $15-40 \%$ e a mortalidade operatória gira em menos de $5 \%$ nos centros de referência.

A baixa eficácia da esofagectomia em termos curativos é explicada, em parte, pela disseminação sistêmica precoce do câncer de esôfago devido a histologia característica do órgão. Assim geralmente os pacientes já apresentam metástases ocultas no momento do diagnóstico. Outro motivo é a relação anatômica intrínseca do esôfago que está próximo a estruturas vitais como a veia cava superior, a aorta e as vias aéreas superiores (estruturas que não podem ser ressecadas para se obter cirurgia R0) ${ }^{11,12}$.

Dado à baixa efetividade da ressecção cirúrgica isolada, é de se esperar que outras modalidades terapêuticas tenham sido desenvolvidas, geralmente em combinação com a cirurgia. Estas associações envolvem radioterapia, quimioterapia ou quimiorradioterapia, podendo ser neoadjuvante e/ou adjuvante. Existe ainda o tratamento combinado com quimiorradioterapia radical exclusiva (sem tratamento cirúrgico) $)^{13,14}$.

A combinação exclusiva de radioterapia com quimioterapia (quimiorradiação) é hoje aceita como tratamento primário das lesões esofágicas localmente avançadas (T3 e T4) e nos pacientes com contra-indicação clinica ao ato operatório. Os benefícios da quimiorradiação sobre a radioterapia isolada estão documentados em estudos clínicos randomizados e controlados ${ }^{15,16}$. A sobrevida média de 12-18 meses e de $15 \%$ em cinco anos têm sido relatada na literatura ${ }^{17}$ séries comparativas não-randomizadas sugerem que a quimioradioterapia é equivalente, se não superior, à ressecção cirúrgica isolada em termos de sobrevida a longo prazo para os tumores avançados ${ }^{18,19}$. Não encontramos ensaios clínicos randomizados comparando a quimioradiação exclusiva com a operação isolada nestes casos.

A esofagectomia de resgate surgiu com o advento da quimiorradiação exclusiva e sua inerente falha locorregional (podendo chegar em até $60 \%$ em algumas séries). É reconhecida a alta morbimortalidade deste procedimento principalmente quando comparada à esofagectomia após neoadjuvância, que requer dose menor de radioterapia aplicada e um menor intervalo de tempo pré-operatório entre o término do tratamento combinado e a ressecção cirúrgica (4-6 semanas). No resgate cirúrgico, a dose de radioterapia aplicada é maior e o intervalo de tempo após o final da radioterapia é indefinido. Isto ocasiona maior grau de fibrose e aderências entre as estruturas periesofagianas com consequiente aumento da dificuldade técnica na dissecção do esôfago, maior índice de lesões iatrogênicas e morbimortalidade operatória.

Em 2002, o MD-Anderson Cancer Center (MDACC) publicou importante estudo que se constituiu numa série 13 pacientes submetidos à esofagectomia de resgate pósquimiorradioterapia exclusiva. Ultilizando-se um controle heterogêneo de 99 pacientes submetidos à terapia bimodal neoadjuvante, encontraram maior morbimortalidade operatória do resgate cirúrgico quando compararam com a cirurgia após neoadjuvância e sobrevida em cinco anos de $25 \%{ }^{20}$.

Nakamura et al. publicaram em 2004 uma série de 27 pacientes submetidos ao resgate cirúrgico de carcinoma epidermóide de esôfago após quimiorradiação exclusiva e também compararam-na a um grupo controle submetido a neoadjuvância (28 casos). Em nove casos, a ressecção foi R1 ou R2. Ao contrário do trabalho do MDACC, esta publicação não encontrou diferença em termos de morbi-mortalidade operatória e sobrevida em relação aos dois grupos ${ }^{21}$.

Em janeiro de 2005, foi publicado um estudo prospectivo não-randomizado avaliando 53 pacientes quanto à necessidade de esofagectomia após quimiorradioterapia radical exclusiva. Nesta série, para os respondedores ao tratamento combinado a esofagectomia não acrescentou benefícios. No entanto, para os pacientes que não responderam à quimiorradiação, a ressecção cirúrgica aumentou a sobrevida em cinco anos 22

De fato, é reconhecida uma maior morbimortalidade da esofagectomia pós-quimiorradioterapia (neoadjuvante ou exclusiva) em relação aos pacientes virgens de tratamento. Fístulas e deiscência anastomótica, síndrome de desconforto respiratório do adulto (SARA) e complicações relacionadas às vias aéreas são mais comuns nos casos de pacientes submetidos a quimioradiação pré-operatória. Centros especializados de referência relatam mortalidade de aproximadamente 10\% (o dobro dos pacientes virgens de tratamento $)^{23,24}$.

As duas maiores complicações da esofagectomia de resgate são relacionadas com a anastomose e a função pulmonar, o que literalmente foi confirmado na nossa série. Outras complicações relatadas especificamente ao resgate cirúrgico são: necrose e fístula de vias aéreas, injúria do nervo laríngeo recorrente, quilotórax e derrame pericárdico. Todas estas complicações estão relacionadas a uma maior dose de radiação aplicada sobre o mediastino. 
Tivemos um caso de deiscência e um caso de fístula anastomótica esôfago-gástrica, um caso de fístula esôfagotraqueal cervical, além de cinco casos de pneumonia, o que confirma os dados acima referidos. Na Tabela 2, comparamos os nossos resultados com dois grandes e mais recentes estudos publicados até então, sendo um da escola americana ( $M D$ Anderson Cancer Center) e outro da escola japonesa (Tokyo Women's Medical University).

Como demonstramos, mesmo em centros de referência especializados no tratamento do câncer de esôfago, a amostragem das séries ainda é bastante restrita. A primeira esofagectomia de resgate procedida no INCa foi em 1999, sendo a maioria realizada recentemente (2004-2006), daí nosso curto follow-up médio apresentado (12,2 meses).

A quimiorradioterapia exclusiva no câncer de esôfago tem ganhado bastante popularidade nos últimos anos, principalmente nos casos loco-regionalmente avan- çados (T3 ou T4) e naqueles pacientes sem reserva fisiológica para a esofagectomia (Performace status, três ou quatro ou co-morbidades incapacitantes). De fato, muitos pacientes recuperaram o estado geral após término do tratamento combinado, tornando-os aptos à ressecção cirúrgica.

Assim a esofagectomia de resgate demonstrou-se factível tecnicamente, porém apresenta alta morbidade, constituindo-se na única possibilidade de cura para os casos de recidiva ou persistência de doença pós-quimioradiação exclusiva. Representa, portanto a melhor segunda linha de tratamento na falha local após quimiorradiação exclusiva, embora ainda necessite de maiores estudos para sua completa validação. No INCa, a confecção de protocolos para um estudo prospectivo já está em andamento, podendo-se no futuro, avaliar os resultados da esofagectomia de resgate com maior grau de exatidão.

Tabela 2 - Comparação dos resultados do INCa com escola japonesa e americana.

\begin{tabular}{lccl}
\hline & MDACC*(2002) & TWMU**(2004) & INCa/Brasil (2006) \\
\hline No de casos & 13 & 27 & 14 \\
Tempo cirúrgico (mediana) & $542 \mathrm{~min}$ & $312 \mathrm{~min}$ & 305 min $(240-430)$ \\
Tempo de UTI(mediana) & $12,2 \mathrm{dias}$ & $5,9 \mathrm{dias}$ & 4 dias $(2-37$ dias) \\
Taxa de Fístula & $05 \quad(38 \%)$ & $06 \quad(22 \%)$ & $02(15,3 \%)$ \\
Tempo de internação(mediana) & $29,4 \mathrm{dias}$ & $39,9 \mathrm{dias}$ & 14,4 dias (11-59 dias) \\
Morbidade geral & $77 \%$ & $\mathrm{NR} * * *$ & $69,2 \%$ \\
Mortalidade operatória & $02 \quad 01 \quad(3,7 \%)$ & 0 \\
Ressecção Ro & $\mathrm{NR}$ & $18 \quad(67 \%)$ & $13(92,8 \%)$ \\
Seguimento médio & 27 meses & $\mathrm{NR}$ & 12,2 meses \\
Sobrevida média & $\mathrm{NR}$ & $\mathrm{NR}$ & 12,6 meses \\
\hline
\end{tabular}

*MD-Anderson Cancer Center

** Tokyo Women's Medical University

***Não relatado

\begin{abstract}
Objective: To present the experience and the results from the Abdominopelvic Surgery Section from the National Cancer Institute in the rescue esophagectomy in patients previously submitted to the exclusive chemoradiotherapy as primary treatment. Methods: 14 patients submitted to the esophagectomy of rescue from March of 1999 to May of 2006 were analyzed retrospectively. Results: The median age was of 63 years (39-72 years). Eight patients were females and six were males. Nine patients presented the tumor in the medium esophagus and five patients presented the disease in the distal esophagus; epidermoid carcinoma occurred in 12 patients and adenocarcinoma in two patients. The median of the surgical time was of 305 minutes (240-430 minutes). Operation with complete resection (RO) of the tumor was performed in 13 patients and one had metastatic residual disease to lung apices. Total morbidity was of $69.2 \%$ and there was no operative mortality. All patients were discharged. Conclusion: The esophagectomy of rescue was shown to be technically viable; however, it presents a high operative morbidity. It represents the best treatment option for the cure of recurrent tumor, or persistent tumor after exclusive radical chemoradiotherapy. All therapies are considered palliatives, with disappointing survival results.
\end{abstract}

Key words: Esophagectomy; Esophageal neoplasms; Combined modality therapy; Chemotherapy; Radiotherapy; Health Facilities.

\section{REFERÊNCIAS}

1. Brasil. Ministério da Saúde. Instituto Nacional de Câncer. Estimativas de incidência e mortalidade, 2003. Rio de Janeiro: INCa; 2003. 94 p.
2. Brown LM, Devesa SS. Epidemiologic trends in esophageal and gastric cancer in the United States. Surg Oncol Clin N Am. 2002;11(2):235-56.

3. Crew KD, Neugut AI. Epidemiology of upper gastrointestinal malignancies. Semin Oncol. 2004;31(4):450-64. 
4. Stein HJ, Brucher BL, Sendler A, Siewert JR. Esophageal cancer: patient evaluation and pre-treatment staging. Surg Oncol. 2001;10(3):103-11.

5. Gamliel Z, Krasna MJ. Multimodality treatment of esophageal cancer. Surg Clin North Am. 2005 85(3):621-30.

6. Penman ID, Henry E. Advanced esophageal cancer. Gastrointest Endosc Clin N Am. 2005;15(1):101-16.

7. Swisher SG, Deford L, Merriman KW, Merrimax KW, Walsh GL, Smythe R, Vaporicyan A, Ajani JA, brown T, Komaki R, Roth JA, Putnam JB. Effect of operative volume on morbidity, mortality, and hospital use after esophagectomy for cancer. J Thorac Cardiovac Surg. 2000;119(6):1126-35.

8. Lerut T. The surgeon as a prognostic factor. Ann Surg. 2000;232(6):729-32.

9. Urba S. Combined modality therapy of esophageal cancer standard of care? Surg Oncol Clin N Am. 2002;11(2):377-86.

10. Urschel JD, Ashiku S, Thurer R, Sellke FW. Salvage or planned esophagectomy after chemoradiation therapy for locally advanced esophageal cancer-a review. Dis Esophagus. 2003;16(2):60-5.

11. Patel AM, Buenaventura PO. Current staging of esophageal carcinoma. Surg Clin North Am. 2005;85(3):555-67.

12. Nishimaki T, Shimoji H, Sunagawa H. Recent changes and the future roles of esophageal cancer surgery. Ann Thorac Cardiovasc Surg. 2004;10(6):324-32.

13. Das P, Fukami N, Ajani JA. Combined modality therapy of localized gastric and esophageal cancers. J Natl Compr Canc Netw. 2006;4(4):375-82.

14. Mooney MM. Neoadjuvant and adjuvant chemotherapy for esophageal adenocarcinoma. J Surg Oncol. 2005;92(3):230-8.

15. Herskovic A, Martz K, al-Sarraf M, Leichman L, Brindle J, Vaitkevicius V, Cooper J, byhardt R, davis L, Emami B. Combined chemotherapy and radiotherapy compared with radiotherapy alone in patients with cancer of the esophagus. N England J Med. 1992;326(24):1593-8.

16. Rebecca WO, Richard MA. Combined chemotherapy and radiotherapy (without surgery) compared with radiotherapy alone in localized carcinoma of the esophagus. Cochrane Database Syst Rev. 2003;(1):CD002092. Review. Update in: Cochrane Database Syst Rev. 2006;(1):CD002092.

17. Minsky BD. Carcinoma of the esophagus. Part 1: Primary therapy. Oncology. 1999;13(9):1225-32, 1235-6.

18. Chan A, Wong A. Is combined chemotherapy and radiation therapy equally effective as surgical resection in localized esophageal carcinoma? Int J Radiat Oncol Biol Phys. 1999;45(2):265-70.
19. Murakami M, Kuroda Y, Nakajima T, Okamoto Y, Mizowaki T, Kusumi F, Hajiro K, Nishimura S, Matsusue S, Takeda H. Comparison between chemoradiation protocol intended for organ preservation and conventional surgery for clinical T1-T2 esophageal carcinoma. Int J Radiat Oncol Biol Phys. 1999;45(2):277-84.

20. Swisher SG, Wynn P, Putnam JB, Mosheim MB, Correa AM, Komaki RR, Ajani JA, Smythe WR, Vaporciyan AA, Roth JA, Walsh GL. Salvage esophagectomy for recurrent tumors after definitive chemotherapy and radiotherapy. J Thorac Cardiovasc Surg. 2002;123(1):175-83.

21. Nakamura T, Hayashi K, Ota M, Eguchi R, Ide H, Takasaki K, Mitsuhashi N. Salvage esophagectomy after definitive chemotherapy and radiotherapy for advanced esophageal cancer. Am J Surg. 2004;188(3):1261-6.

22. Fujita H, Sueyoshi S, Tanaka T, Tanaka Y, Matono S, Mori N, Shirouzu K, Yamana H, Suzuki G, Hayabuchi N, Matsui M. Esophagectomy: is it necessary after chemoradiotherapy for a locally advanced T4 esophageal cancer? Prospective nonrandomized trial comparing chemoradiotherapy with surgery versus without surgery. World J Surg. 2005;29(1):2530; discussion 30-1.

23. Urshel JD, Sellke FW. Complications of salvage esophagectomy. Med Sci Monit. 2003;9(7):RA173-80.

24. Pinto CE, Pinheiro RN, Kesley R. Morbidity and mortality after esophagectomy of cancer in Brazilian National Cancer Institute [abstract]. Dis Esophagus. 2004;17 suppl 1:A149.

Como citar este artigo:

Pinto CE, Dias JA, Sá EAM, Carvalho ALL. Esofagectomia de resgate após quimiorradioterapia radical exclusiva: resultados do departamento de cirurgia abdôminopélvica do instituto nacional de câncer. Rev Col Bras Cir. [periódico na Internet] 2007;34(1). Disponível em URL: http://www.scielo.br/rcbc

Endereço para correspondência:

Carlos Eduardo Pinto

Av.Canal de Marapendi,2915/105 B.3

Barra da Tijuca

22631-050 - Rio de Janeiro - RJ

E-mail: caudu2003@yahoo.com.br 